\title{
60 Jahre Weltärztebund - ein kurzes Zwischenresümee
}

\section{Markus Rudolphi}

Cons. social-medical and financial affairs, World Medical Association
Korrespondenz:

Markus Rudolphi, MD

c/o Bundesärztekammer

Herbert-Lewin-Platz 1

D-10623 Berlin

markus.rudolphi@baek.de
Der Weltärztebund (World Medical Association WMA) ist eine der massgeblichen internationalen Organisationen im Bereich der Gesundheitsversorgung. Er wurde im September 1946 von Ärztevertretern aus 29 Staaten als Nachfolgeorganisation der Association Professionnelle Internationale des Médecins (APIM) gegründet, die die ärztlichen Interessen in den Jahren 1926 bis 1946 vertrat. Die erste Generalversammlung des Weltärztebundes fand nach Satzungslegung am 18. September 1947 in Paris statt.

In den nachfolgenden Jahren wurden durch den Weltärzteverband richtungweisende Positionen verabschiedet:

- 1948/1949: Deklaration von Genf (Genfer Gelöbnis), 1. Internationale Berufsordnung;

- 1964: Deklaration von Helsinki (sie bildet bis heute einen wesentlichen Grundstein eines gemeinsamen «Code of Ethics», Überarbeitung und Anpassung 1975 und 2000);

- 1975: Deklaration von Tokio (Richtlinie für Ärzte bei Folterung, Grausamkeit und anderen unmenschlichen oder die Menschenwürde verletzenden Handlungen in Verbindung mit Haft und Gefangenschaft; Anpassung 2006);

- 1981: Deklaration von Lissabon (Stärkung der Patientenrechte).

Diese Deklarationen stellen nur einen Ausschnitt der vielen Bemühungen und Positionierungen des Weltärztebundes in den letzten 60 Jahren dar. Im Laufe der Jahre haben sich die Arbeitsbereiche, in denen der Weltärztebund tätig ist, vervielfacht. Derzeit umfassen sie unter anderem die Konsensbildung und Entwicklung von Leitlinien im Bereich der ärztlichen Ethik, relevante sozialmedizinische Fragen sowie die Vertretung der Interessen der Ärzteschaft in internationalen Organisationen wie z. B. den Vereinten Nationen (UN), der Weltgesundheitsorganisation (WHO), der UNESCO oder dem UNICEF. Zudem unterstützt der Weltärztebund auf Basis einzelner Anfragen regionale und nationale Ärzteverbände bei ihren spezifischen Anliegen. Ebenso stehen wie in Deutschland - die Stärkung der ärztlichen Selbstverwaltung und deren zukünftige strategi- sche Ausrichtung als ein wesentlicher Aspekt auf der Agenda.

Der Weltärztebund gliedert sich in eine jährlich tagende Generalversammlung, einen Vorstand, unterschiedliche Ausschüsse sowie einen bedarfsmässig einberufenen Exekutivausschuss. Die Generalversammlung wählt in jedem Jahr einen neuen Präsidenten. Für die Legislaturperiode 2007/2008 fiel die Wahl einstimmig auf Dr. Jon Snaedle (Island). Der vergleichsweise enge zeitliche Turnus soll eine möglichst breite Beteiligung der unterschiedlichen nationalen Ärzteorganisationen sicherstellen.

Generalsekretär des Weltärztebundes ist seit 2004 Dr. Dr. h.c. Otmar Kloiber. Die letzte Generalversammlung des Weltärztebundes fand im Oktober 2007 in Kopenhagen statt. Neben der Weiterentwicklung und Aktualisierung der massgeblichen Positionen des Weltärztebundes, wie z. B. der Deklaration von Helsinki, standen aktuelle Themen wie die Telemedizin oder die Verabschiedung einer Resolution zum Nikotinmissbrauch auf dem Programm. Zudem wurde die kritische Situation der Menschenrechte in Zimbabwe erörtert sowie die ärztliche Verantwortung bei der Dokumentation und Denunziation von Folterungen oder entwürdigenden Behandlungen diskutiert.

\section{Engagement für Menschenrechte}

$\mathrm{Zu}$ den regelmässigen Aktivitäten des Weltärztebundes im Bereich der Menschenrechte zählen unter anderem die Durchführung eines Kurses zur Anwendung eines Protokolls der Vereinten Nationen zur besseren Untersuchung von Foltervorfällen («Istanbul-Protokoll») sowie individuelle Interventionen als Reaktion auf aktuelle Menschenrechtsverletzungen.

Wie erfolgreich die zeitweise hartnäckigen Interventionen des Weltärztebundes sind, zeigt das aktuelle Beispiel der Organentnahmen und -transplantationen in China, wo bis vor kurzem noch Anhängern der Falun-Gong-Bewegung systematisch Organe entnommen und an zahlungskräftige Ausländer verkauft wurden. Auch wenn zur Wahrung des erzielten Erfolges hier auf eine Detaildarlegung verzichtet wird, konnten durch 
die wiederholten Gespräche mit dem Gesundheitsministerium in Peking Änderungen durchgesetzt werden, die noch vor einigen Jahren wenn nicht sogar vor Monaten - undenkbar gewesen sind.

Der Weltärztebund integriert seine Aktivitäten in die World Health Professions Alliance (WHPA). Diese weltweit aufgestellten Organisationen repräsentieren den Weltverband der Krankenschwestern, der Apotheker, der Zahnärzte sowie der Ärzte. Die Allianz ermöglicht die Bündelung des Wissens und der Erfahrung von etwa 23 Millionen im Gesundheitssystem Beschäftigten bei den Debatten mit Regierungen, politischen Entscheidungsträgern oder der WHO. Der Weltärztebund unterstützt die gemeinsamen Anliegen innerhalb dieser Allianz durch die Ausrichtung von Seminaren oder öffentlichen Informationsveranstaltungen wie z. B. zu der drastisch zunehmenden Quote an Medikamentenfälschungen, zur Nikotinmissbrauchsbekämpfung, HIV/AIDS oder aber auch der Stigmatisierung infolge psychiatrischer Behandlungen.

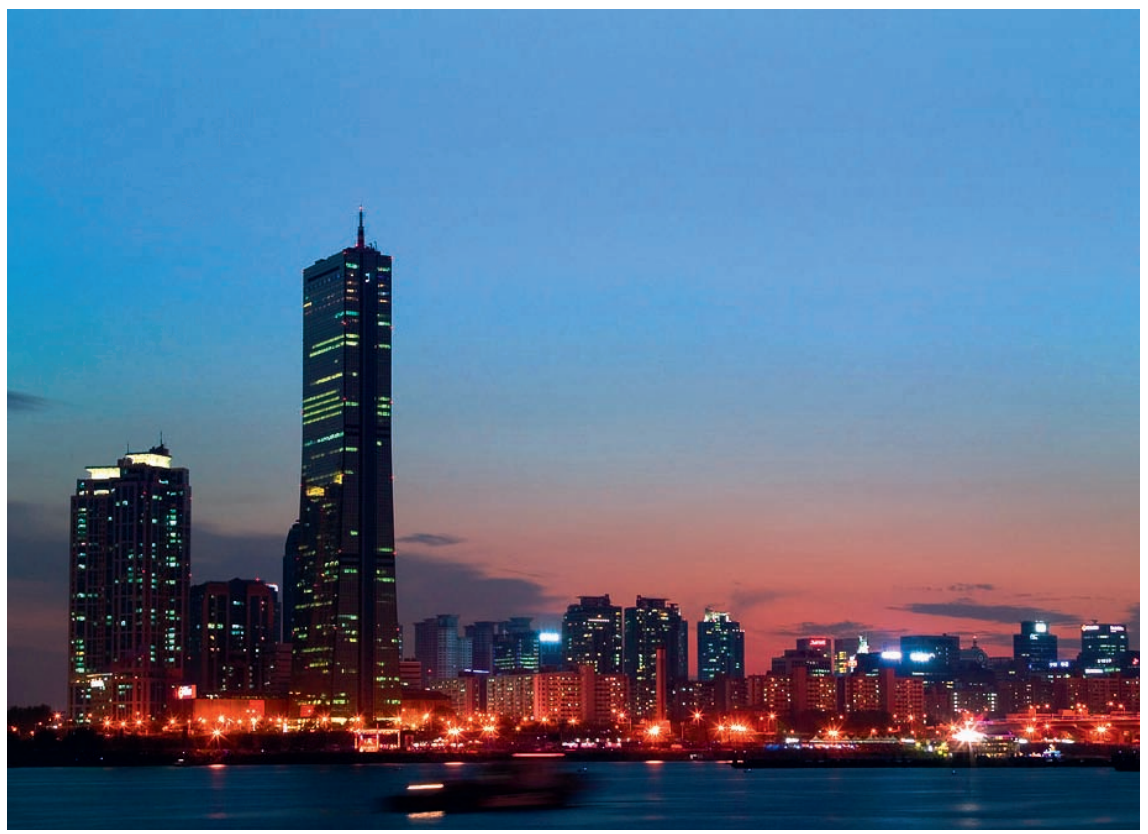

Die 59. Generalversammlung des Weltärzteverbandes findet vom 15. bis 18. Oktober 2008 in Seoul, Südkorea, statt.
Neben den gemeinsamen Bemühungen innerhalb der Allianz ist der Weltärztebund auch im Bereich der ärztlichen Fortbildung aktiv.

Im Fokus stehen derzeit Themen wie:

- ärztliche Ethik;

- ärztliche Versorgung von Gefangenen;

- Unterstützung und Durchführung eines Kursus zu der Behandlung von Patienten mit multiresistenter Tuberkulose.

Die ethischen Grundlagen ärztlichen Handelns sind vom Weltärztebund in einem «Medical Ethics Manual» zusammengefasst worden. Sie bilden die Basis für ein weltweites gemeinsames ethisches Verständnis im Sinne eines «Code of Ethics». Interessierten steht dieses Manual als kostenloser Download unter www.wma.net zur Verfügung.

\section{Zugang zu Innovationen von zentraler Bedeutung}

Die künftigen Herausforderungen für den Weltärztebund sind vielfältig. Zu den ebenso wichtigen wie kontrovers zu diskutierenden Themen gehören die zukünftige Ausrichtung von Berufsordnungen, die Diskussion zum Verständnis über den Anfang und das Ende menschlichen Lebens, die weitere Revision der Deklaration von Helsinki, die Handhabung epidemiologischer Forschung, aber auch der weitere Umgang mit dem Thema Organtransplantation. Darüber hinaus werden sozialmedizinische Themen wie die Tabakbekämpfung, der Alkoholmissbrauch oder die sogenannte «disaster preparedness» eine wesentliche Rolle spielen. Der Zugang zu Innovationen in Diagnostik und Therapie sowie deren Implementierung ist vor allem in weniger wohlhabenden Ländern ein Thema von grosser Bedeutung. Im Sinne der ärztlichen Interessenvertretung werden auch die Themen Ärztemangel und -migration, Arbeitsbedingungen von Ärzten, Kompetenzverteilungen (task shifting, scope of practice) und die Zukunft der ärztlichen Selbstverwaltung zunehmend relevant sein. Es bleibt zu hoffen, dass ...

Informationen zum Weltärztebund und dessen Aktivitäten finden Sie unter www.wma.net. 